\begin{tabular}{|c|l|}
\hline Title & ח - hydrogen bonding between water and aromatic hy drocarbons at high temperatures and pressures \\
\hline Author(s) & Furutaka, Seiya; Ikawa, Shun-ichi \\
\hline Citation & $\begin{array}{l}\text { The Journal of Chemical Physics, 117(2), 751-755 } \\
\text { https://doi.org/10.1063/1.1484105 }\end{array}$ \\
\hline Issue Date & 2002 \\
\hline Doc URL & http://hdl.handle.net/2115/1405 \\
\hline Rights & Copyright $\odot 2002$ A merican Institute of Physics \\
\hline Type & article \\
\hline File Information & JCP117-2.pdf \\
\hline
\end{tabular}

Instructions for use 


\title{
$\pi$-hydrogen bonding between water and aromatic hydrocarbons at high temperatures and pressures
}

\author{
Seiya Furutaka and Shun-ichi Ikawa \\ Division of Chemistry, Graduate School of Science, Hokkaido University, Sapporo 060-0810, Japan
}

(Received 1 March 2002; accepted 17 April 2002)

\begin{abstract}
Infrared $\mathrm{OH}$ stretching absorption of HDO isolated in aromatic hydrocarbons have been measured at temperatures of 473 and $523 \mathrm{~K}$ and at pressures in the 100-350 bar range. The peak frequencies are dependent on the solvents and their order, benzene $>$ toluene $>$ ethylbenzene $>$ cumene $>o$-xylene $\sim m$-xylene $>$ mesitylene, is exactly the same as the order for the ionization potentials of the hydrocarbons. Shifts of the frequencies from that of HDO in hexane, which was measured as a reference at the same temperature and pressure, were analyzed using a charge transfer theory for hydrogen bonding. Distances between the water molecule and a solvent phenyl ring were estimated to be $2.8 \pm 0.1$ and $2.9 \pm 0.1 \AA$ at 473 and $523 \mathrm{~K}$, respectively. These values are consistent with a structure of a water-benzene complex determined by a jet-cooled microwave spectroscopy. These facts suggest that the $\pi$-hydrogen bond between water and aromatic hydrocarbons exists even at the high temperatures under pressure. (C) 2002 American Institute of Physics.
\end{abstract}

[DOI: $10.1063 / 1.1484105]$

\section{INTRODUCTION}

It has been known for a long time that aromatic hydrocarbons act as hydrogen bonding bases. This tendency was initially confirmed by infrared spectroscopy. ${ }^{1}$ The spectrum of $o$-biphenylol (2-hydroxybiphenyl) in a dilute $\mathrm{CCl}_{4}$ solution showed a doublet in the region of the first overtone of the $\mathrm{OH}$ stretching. The doublet was assigned to two different conformers in equilibrium produced by coupling of the hydroxyl group with the nearby phenyl ring. After that, many researchers have studied intra- or intermolecular interactions that aromatic hydrocarbons act as hydrogen bonding bases. ${ }^{2,3}$ On the other hand, hydrogen bonding of water with benzene was indirectly confirmed by a ${ }^{1} \mathrm{H}-\mathrm{NMR}$ method. ${ }^{4-6}$ The hydrogen bond interaction usually shifts a proton resonance signal to lower fields, but the interaction with benzene shifts the proton signal of water to higher fields. This opposite shift has been interpreted in terms of the diamagnetic anisotropy of the aromatic $\pi$ electrons. The hydrogen bonding interaction of water with aromatic hydrocarbons in the liquid state was also studied by infrared spectroscopy. ${ }^{7-9}$ Furthermore, complexation of water with alkyl-substituted benzenes was studied in argon matrices by infrared spectroscopy. ${ }^{10}$ Comparison of the peak wave numbers of water bands of the complexes and isolated water indicated $\pi$-hydrogen bond interactions between a water molecule and a phenyl ring. The ground-state microwave spectroscopy ${ }^{11}$ and the resonant iondip infrared spectroscopy ${ }^{12,13}$ of jet-cooled water-benzene mixture have revealed gas-phase structure of the waterbenzene complex, in which the hydrogen atom of a water molecule points towards the benzene ring. A $b$ initio calculations have shown that the order of magnitude of the binding energy of the water-benzene complex is 7-12 $\mathrm{kJ} \mathrm{mol}^{-1},{ }^{11,14-16}$ which is intermediate between the binding energy of van der Waals complexes and that of typical hydrogen-bonded complexes. Some authors claimed that the rather large mutual solubility of water with aromatic hydrocarbons as compared with alkanes at ambient condition should be the result of a hydrogen-bondlike interaction between water and $\pi$ electrons of an aromatic ring. ${ }^{17,18}$

This type of interaction involving $\pi$ electrons has been considered to be important in biological systems. ${ }^{19}$ The common constituents of globular proteins such as phenylalanine, tryptophan, and tyrosine possess an aromatic side-chain. When they encounter water molecules in internal hydrophobic cavities of the protein, $\pi$-hydrogen bonds may contribute to stabilize the local structure of the proteins. ${ }^{20}$ On the other hand, if they lie on the surface of proteins, $\pi$-hydrogen bonds will contribute hydration energy. ${ }^{21}$ Water at high temperatures and pressures, that is, the hydrothermal conditions, is prevalent in geochemistry and may have contributed to the origin of life. $^{22,23}$ To understand microscopic mechanism of the supercritical water oxidation of organic wastes, molecular dynamics simulations have been performed. ${ }^{24-26}$ It has been pointed out that a benzene molecule can be preferably approached by the hydrogen atom of a water molecule under the supercritical condition. ${ }^{26}$ The hydrogen bonds between water and aromatics at high temperatures and pressures, however, still remain largely unknown. In the previous papers, ${ }^{27-31}$ we have reported the infrared spectra of HDO in some aromatic hydrocarbons at high temperatures and pressures, and found a certain correlation between the frequency shifts of HDO and the ionization potentials of the aromatic hydrocarbons at $373-473 \mathrm{~K}$ and 100 bar. $^{28}$ This fact suggests that the interaction of water and aromatic hydrocarbons in the high temperature-pressure mixtures can be described as $\pi$-hydrogen-bonding. In order to obtain further insight into the $\pi$-hydrogen-bonding, this paper deals with measurements of the infrared spectra of HDO in aromatic hydrocarbons at the extended range of temperature and pressure and reports 


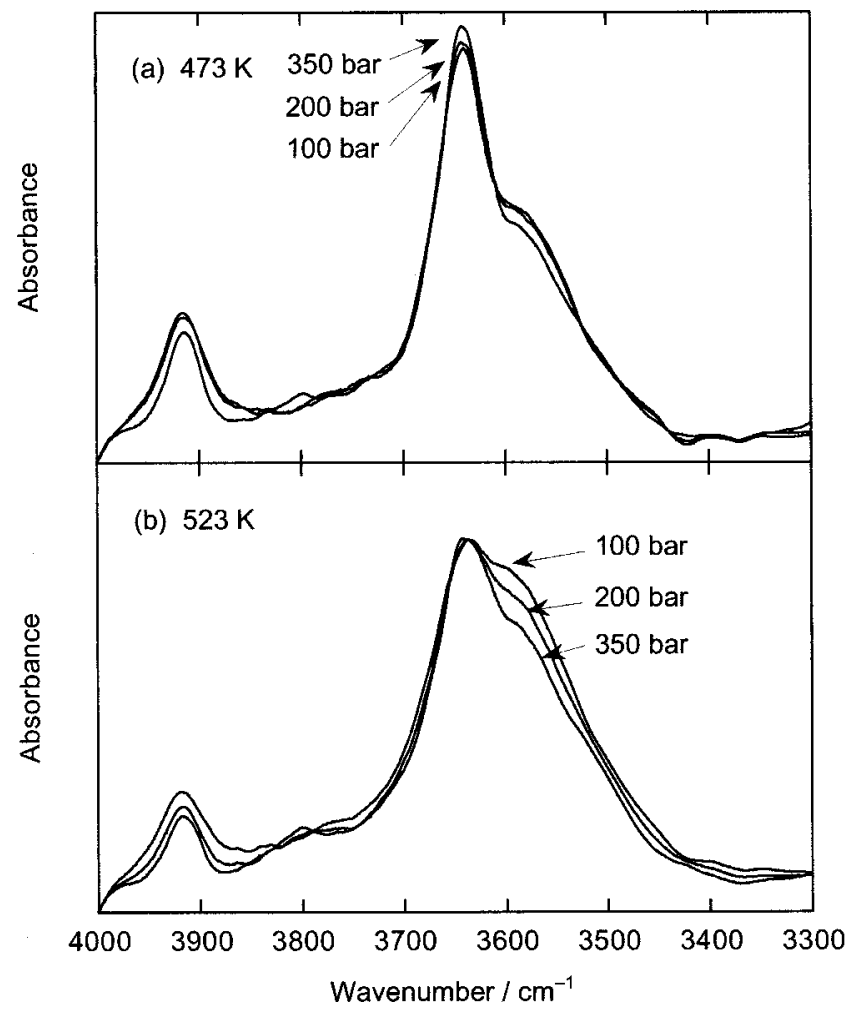

FIG. 1. Infrared absorption of HDO in cumene at $473 \mathrm{~K}$ (a) and $523 \mathrm{~K}$ (b) at a few pressures.

an analysis of the frequency shifts on the bases of a chargetransfer model.

\section{EXPERIMENT}

A high-pressure cell used has been described in detail elsewhere. ${ }^{30,31}$ The optical path length of a sample was 1 $\mathrm{mm}$. Spectroscopic grade benzene, toluene, ethylbenzene, cumene, $o$-xylene, $m$-xylene, mesitylene, and hexane from Nakarai Tesque (Japan) and deuterium oxide $(99.9 \%$ D) from CEA (France) were used as received. The water specimens were mixtures of $\mathrm{H}_{2} \mathrm{O}$ and $\mathrm{D}_{2} \mathrm{O}$ with a ratio of 1:10 and 1:20. Then the ratios of the isotopic species $\mathrm{H}_{2} \mathrm{O}, \mathrm{HDO}$, and $\mathrm{D}_{2} \mathrm{O}$ in these specimens were estimated to be 1:20:100 or 1:40:400. Therefore, contribution of $\mathrm{H}_{2} \mathrm{O}$ to the infrared $\mathrm{OH}$ stretching absorption can be approximately neglected as compared with that of HDO, and the observed $\mathrm{OH}$ band is assigned only to HDO. A syringe pump of liquid chromatography was used for transmitting compressed liquids of hydrocarbons into the cell in which a certain amount of the water specimens had been put in advance and its level adjusted so as to be slightly below the optical path. Infrared absorption of the hydrocarbon-rich phase was observed with a BOMEM DA3 Fourier-transform spectrometer equipped with a $\mathrm{CaF}_{2}$ beamsplitter and a mercury-cadmium-telluride detector. The spectral measurements were performed with 2 $\mathrm{cm}^{-1}$ resolution at sample temperatures 473 and $523 \mathrm{~K}$ and pressures in the 100-350 bar range. The phase equilibrium of the mixture was confirmed by the spectrum that was unchanged for at least $1 \mathrm{~h}$. Absorbance of $\mathrm{HDO}$ in the $\mathrm{OH}$

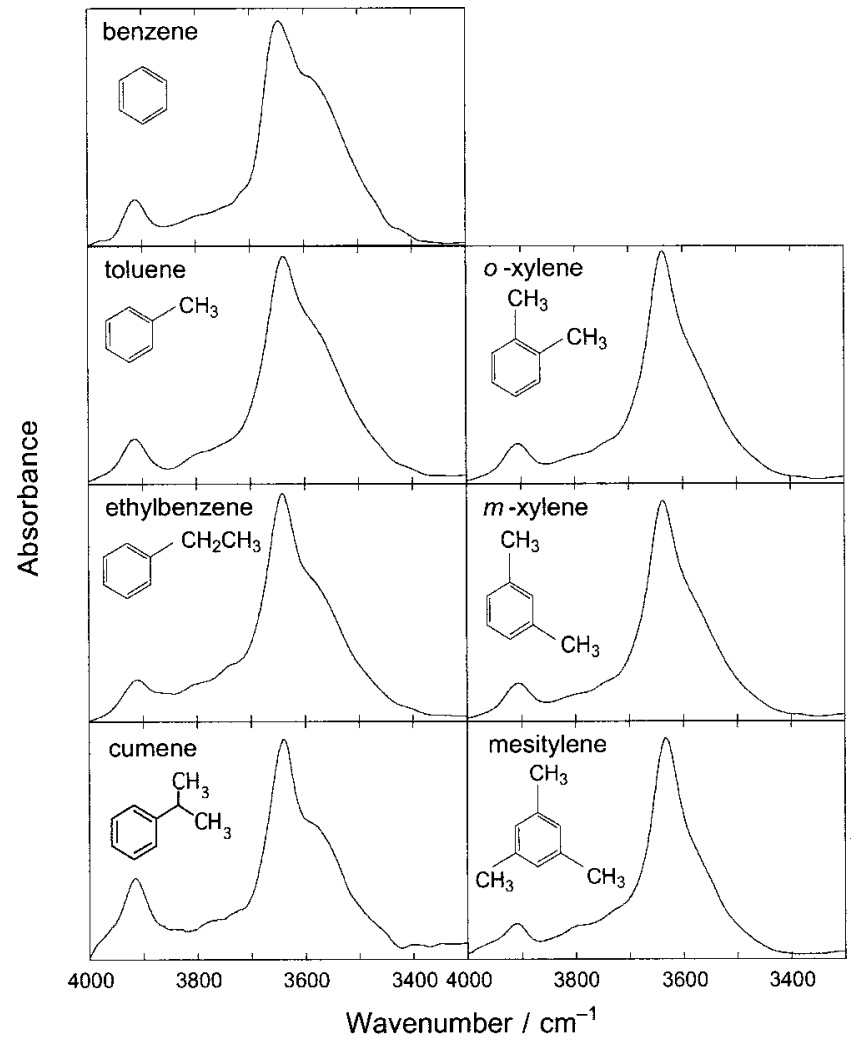

FIG. 2. Infrared absorption of HDO in various alkyl-substituted benzenes at $473 \mathrm{~K}$ and 100 bar.

stretching region was obtained by subtracting the spectrum of neat hydrocarbon measured at the same condition.

\section{RESULTS AND DISCUSSION}

Figure 1 shows the $\mathrm{OH}$ stretching absorption of HDO dissolved in cumene at 473 and $523 \mathrm{~K}$ at a few pressures. Bands at about $3640 \mathrm{~cm}^{-1}$ and $3580 \mathrm{~cm}^{-1}$ are assigned to hydrogen-bond-free $\mathrm{OH}$ groups and hydrogenbonded $\mathrm{OH}$ groups, respectively. ${ }^{27,28}$ It should be mentioned here that the term "hydrogen-bonded" means "hydrogenbonded to water" and the hydrogen-bond-free groups are the ones believed to be bonded to the aromatic hydrocarbons probably through a $\pi$-hydrogen bonding. A band at about $3910 \mathrm{~cm}^{-1}$ is assigned to a combination band of $\mathrm{D}_{2} \mathrm{O} .{ }^{29} \mathrm{In}-$ frared absorption of HDO observed in all the solvents used are very similar in profile as seen in Fig. 2. Positions of the hydrogen-bond-free bands, however, vary with different solvents. The peak frequencies are in the following order, benzene $>$ toluene $>$ ethylbenzene $>$ cumene $>o$-xylene $\sim m$-xylene $>$ mesitylene. This is exactly the same order as that of the vertical ionization potentials ${ }^{32}$ of the hydrocarbons as shown in Fig. 3. The ability of the hydrocarbons as the hydrogen-bond acceptor will be related to their electronrelease tendency which is represented by the ionization potential. Another intriguing point is that the peak frequencies of the monomeric HDO in benzene, $3649-3651 \mathrm{~cm}^{-1}$ in the $473-523 \mathrm{~K}$ range, are near to that of a water-benzene complex, $3650 \mathrm{~cm}^{-1}$, ${ }^{12}$ which was observed for a jet-cooled cluster with resonant ion-dip infrared spectroscopy. This agreement suggests that the monomeric water in benzene at high temperature and pressure undergoes a similar interaction to 


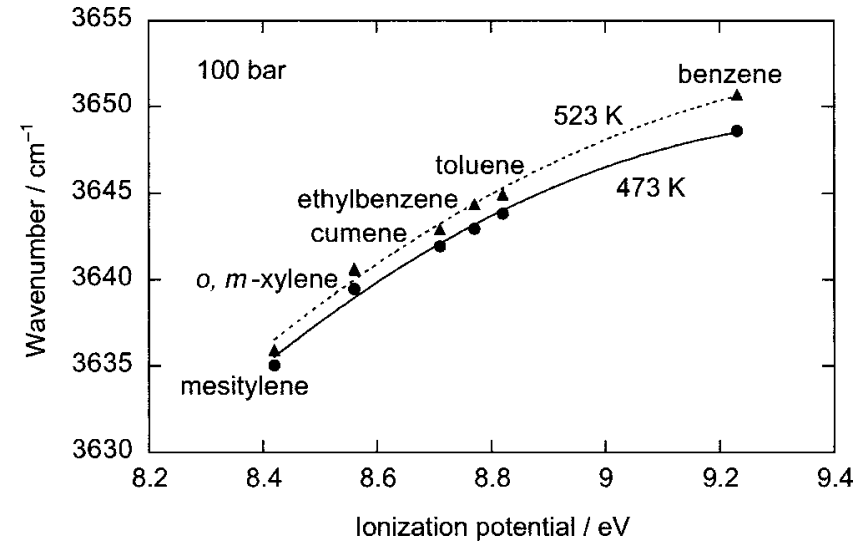

FIG. 3. The correlation between $\mathrm{OH}$ stretching frequencies of HDO and the vertical ionization potential of solvent aromatic hydrocarbons.

that in the low-temperature complex, in which water forms a hydrogen bond with benzene, with one of its hydrogen atoms pointing toward the center of the aromatic ring. ${ }^{11}$ In this configuration, the $\pi$-electron of the benzene ring is thought to act as a hydrogen-bond acceptor.

To characterize further the water-hydrocarbon interaction in the high-temperature-pressure mixtures, we analyzed correlation between the $\mathrm{OH}$ stretching frequencies and the ionization potentials of aromatic hydrocarbons using a charge-transfer model for hydrogen bonding by Ratajczak and Orville-Thomas. ${ }^{33}$ On the basis of Mulliken's secondorder perturbation calculation for loose 1:1 charge-transfer complexes, ${ }^{34}$ they derived the following relationship between the frequency shift of an $\mathrm{X}-\mathrm{H}$ stretching band, $\Delta \nu(\mathrm{X}-\mathrm{H})$, and the vertical ionization potential, $I_{D}$, of an electron donor molecule (aromatic hydrocarbon in the present study)

$$
[\Delta \nu(\mathrm{X}-\mathrm{H})]^{-1}=\frac{a}{\beta_{0}^{2}}\left(I_{D}-E_{A}-C\right),
$$

where, $E_{A}$ is the electron affinity of the electron acceptor molecule (water in the present study), $C$ is the difference in stabilization energies between the excited and ground states, $a$ is a constant for structurally similar hydrogen-bonded complexes, and $\beta_{0}$ is related to the overlap integral between the donor and acceptor orbitals. For convenience, Eq. (1) is rewritten as

$$
[\Delta \nu(\mathrm{X}-\mathrm{H})]^{-1}=m \cdot I_{D}+n,
$$

where

$$
\begin{aligned}
& m=a \cdot \beta_{0}^{-2}, \\
& n=-a \cdot \beta_{0}^{-2} \cdot\left(E_{A}+C\right)=-m \cdot\left(E_{A}+C\right) .
\end{aligned}
$$

Thus, a linear relationship is expected between $(\Delta \nu)^{-1}$ and $I_{D}$.

The frequency shift $\Delta \nu$ is given by $\Delta \nu=\nu_{F}-\nu_{B}$, where $\nu_{F}$ and $\nu_{B}$, respectively, are $\mathrm{OH}$ stretching frequencies free from and influenced by the charge transfer effect. As a value of $\nu_{F}$, the $\mathrm{OH}$ stretching frequency of HDO in hexane at the same temperature and pressure as for $\nu_{B}$ is used. It is assumed that $\nu_{F}$ involves a similar interaction to that in aro-

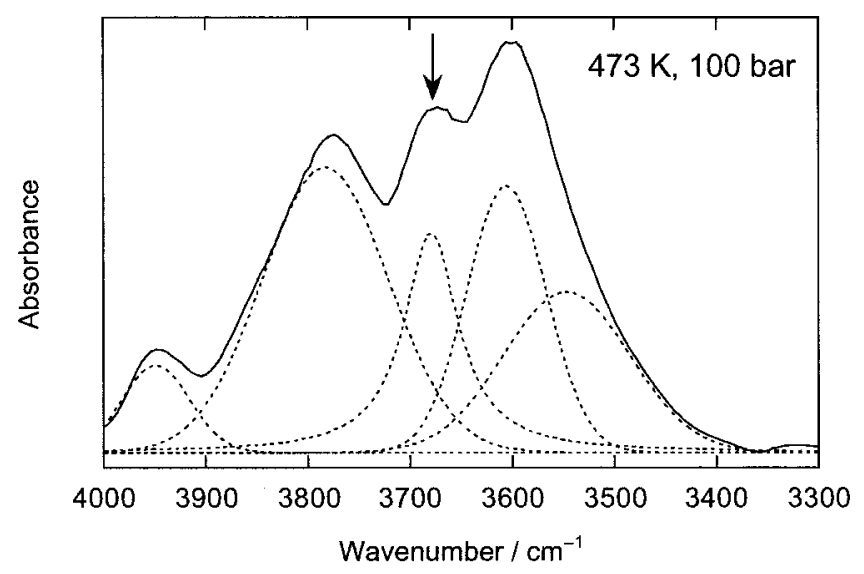

FIG. 4. Infrared absorption of HDO in hexane at $473 \mathrm{~K}$ and 100 bar. The arrow indicates the $\nu_{F}$ band, the Q branch of HDO molecules free from water-water association.

matic hydrocarbons except the charge transfer effect. Figure 4 shows infrared absorption of HDO in hexane at $473 \mathrm{~K}$ and 100 bar. The profile of the $\mathrm{OH}$ absorption is remarkably different from those in aromatic hydrocarbons. The bands at around 3680 and $3600 \mathrm{~cm}^{-1}$ are assigned to the hydrogenbond-free and hydrogen-bonded species by the analogy with the $\mathrm{OH}$ bands in aromatic hydrocarbons. The band at about $3780 \mathrm{~cm}^{-1}$ is assigned to a vibration-rotation transition of hydrogen-bond-free HDO, by analogy with the peak of frequency measured in an argon matrix. ${ }^{35}$ The corresponding bands can be seen in the spectra in Fig. 2, but their relative intensities are significantly smaller than that of $3780 \mathrm{~cm}^{-1}$ band in hexane. This fact indicates that the rotational motion of the water molecule in hexane is significantly free as compared with that in aromatic hydrocarbons, and may be quasifree as in decane and octane at ambient condition. ${ }^{36,37}$ This is probably due to absence of a hydrogen-bondlike interaction between water and hexane, being different from the interaction between water and aromatic hydrocarbons. It should be noted here that the $3780 \mathrm{~cm}^{-1}$ band assigned to the vibration-rotation transition should have a lower frequency counterpart with roughly the same broadening but less intensity. This component can be seen by a band decomposition shown by dotted lines in Fig. 4. Details of the band shape analysis will be reported elsewhere.

The resulting values of $(\Delta \nu)^{-1}$ are plotted against $I_{D}$ in Fig. 5. As expected above, the plots are approximately linear for every experimental condition. This fact indicates that the interaction between water and aromatic hydrocarbons in the mixtures at high temperatures and pressures can be described as the charge-transfer interaction or the $\pi$-hydrogen bonding.

Using the $E_{A}$ value of water, $1.2 \mathrm{eV}^{38}$ the distance $r$ between water and the phenyl ring can be estimated from the experimentally determined $m$ and $n$ values on the assumption that $r$ is independent of the aromatic hydrocarbons used in the present study. From Eqs. (3) and (4), we obtain

$$
\frac{n}{m}+E_{A}=-C .
$$

The stabilization energy of the excited state, or the charge transfer state, is approximately given by the Coulomb energy 


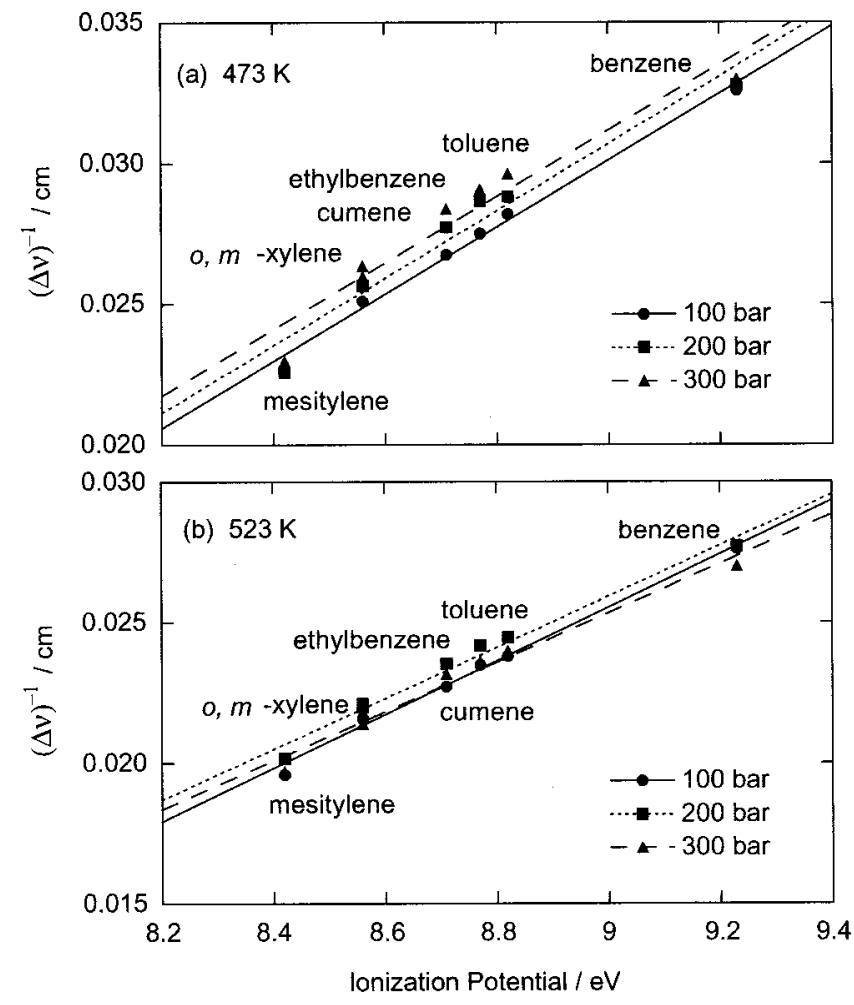

FIG. 5. Plots of the frequency shifts of the $\mathrm{OH}$ stretching of HDO against the ionization potentials of the solvent aromatic hydrocarbons at $473 \mathrm{~K}$ (a) and $523 \mathrm{~K}$ (b) at $100-300$ bar.

of the ion pair and much larger than that of the ground state. $^{39}$ Therefore, $C$ is approximately given by $-q^{2} /\left(4 \pi \epsilon_{0} r\right)$, where $q$ is the elementary electric charge $\left(q=1.602 \times 10^{-19} \mathrm{C}\right)$ and $\epsilon_{0}$ is the electric permittivity of vacuum $\left(\epsilon_{0}=8.854 \times 10^{-12} \mathrm{~F} \mathrm{~m}^{-1}\right)$. Consequently, the distance or, strictly speaking, the charge transfer distance $r$ between water and the aromatic hydrocarbon is given by the following relationship:

$$
r=\frac{q^{2}}{4 \pi \epsilon_{0}\left[-(n / m)-E_{A}\right]} .
$$

Using the values of $n / m$ estimated from the slope and the intercept of the plots in Fig. 5, $r$ is estimated to be $2.8 \pm 0.1$ and $2.9 \pm 0.1 \AA$ at 473 and $523 \mathrm{~K}$, respectively. The $r$ values are almost independent of pressure in the 100-350 bar range. Figure 6 shows a schematic of a water-benzene complex, where a cross $(X)$ represents the center of the benzene ring. In the MP-2 optimized structure, ${ }^{15}$ the distance from $\times$ to the hydrogen $(\mathrm{H})$ involved in the $\pi$-hydrogen bond is $2.302 \AA$, and the distance to the oxygen $(\mathrm{O})$ of a water molecule is $3.210 \AA$, which is in fair agreement with the experimental value of $3.347 \AA$ determined from jet-cooled microwave spectra. ${ }^{11}$ Considering the charge-transfer from the $\pi$-orbital of the aromatic ring to the antibonding orbital of the $\mathrm{OH}$ bond, it seems reasonable that the $r$ values are in the middle of the two explicitly defined distances mentioned above. Therefore, the present experimental result indicates that the concept of $\pi$-hydrogen bonding is effective for describing the water-aromatic hydrocarbon interaction in the hightemperature-pressure mixtures.
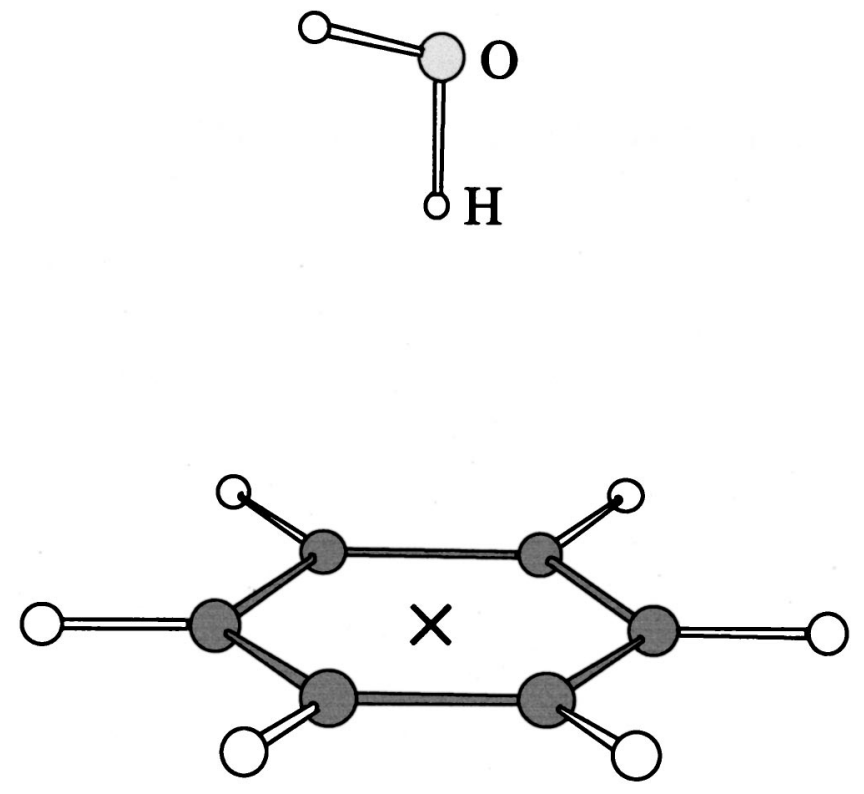

FIG. 6. Schematic of a water-benzene complex.

\section{CONCLUSION}

Infrared spectra of HDO dissolved in various aromatic hydrocarbons have been measured at 473 and $523 \mathrm{~K}$ and pressures in the 100-350 bar range. The $\mathrm{OH}$ stretching frequencies assigned to HDO monomers, which are free from water-water association, are in the following order: benzene $>$ toluene $>$ ethylbenzene $>$ cumene $>0$-xylene $\sim m$-xylene $>$ mesitylene. We have analyzed a correlation of the $\mathrm{OH}$ stretching frequencies with the ionization potentials of the solvent hydrocarbons using a charge transfer theory for hydrogen bonding. The charge transfer distance between the water molecule and the solvent aromatic ring estimated by that model is consistent with the structure of a waterbenzene complex obtained by a jet-cooled microwave spectroscopy ${ }^{13}$ and theoretical calculations. ${ }^{15}$ This fact suggests that the $\pi$-hydrogen bond between water and aromatic hydrocarbons exists even at high temperatures under pressure.

\section{ACKNOWLEDGMENTS}

This work was supported by the Research Fellowships of the Japan Society for the Promotion of Science for Young Scientist to S.F. and by the Grant-in-Aid for Scientific Research from the Ministry of Education, Culture, Sports, Science, and Technology of Japan (No. 13440169).

${ }^{1}$ O. R. Wulf, U. Liddel, and S. B. Hendricks, J. Am. Chem. Soc. 58, 2287 (1936).

${ }^{2}$ G. C. Pimentel and A. L. McClellan, The Hydrogen Bond (Freeman, San Francisco, 1960).

${ }^{3}$ Z. Yoshida and E. Osawa, J. Am. Chem. Soc. 87, 1467 (1965).

${ }^{4}$ A. D. Cohen and C. Reid, J. Chem. Phys. 25, 790 (1956).

${ }^{5}$ M. Baron, Anales. Asoc. Quim. Argentina 67, 203 (1979).

${ }^{6}$ M. Nakahara and C. Wakai, Chem. Lett. 1992, 809.

${ }^{7}$ E. Gentric, A. La Narvor, and P. Saumagne, C. R. Acad. Sci. Paris, Ser. C 270, 1053 (1970).

${ }^{8}$ O. D. Bonner and Y. S. Choi, J. Phys. Chem. 78, 1723 (1974).

${ }^{9}$ J. Cz. Dobrowolski and M. H. Jamróz, J. Mol. Struct. 293, 147 (1993).

${ }^{10}$ A. Engdahl and B. Nelander, J. Phys. Chem. 91, 2253 (1987). 
${ }^{11}$ S. Suzuki, P. G. Green, R. E. Bumgarner, S. Dasgupta, W. A. Goddard III, and G. A. Blake, Science 257, 942 (1992).

${ }^{12}$ R. N. Pribble and T. S. Zwier, Faraday Discuss. 97, 229 (1994).

${ }^{13}$ H.-D. Barth, K. Buchhold, S. Djafari, B. Reimann, U. Lommatzsch, and B. Brutschy, Chem. Phys. 239, 49 (1998).

${ }^{14}$ S. Y. Fredericks, K. D. Jordan, and T. S. Zwier, J. Phys. Chem. 100, 7810 (1996).

${ }^{15}$ D. Feller, J. Phys. Chem. 103, 7558 (1999).

${ }^{16}$ P. Tarakeshwar, H. S. Choi, S. J. Lee, J. Y. Lee, K. S. Kim, T.-K. Ha, J. H. Jang, J. G. Lee, and H. Lee, J. Chem. Phys. 111, 5838 (1999).

${ }^{17}$ S. Goldman, Can. J. Chem. 52, 1668 (1974).

${ }^{18}$ P. Ruelle, M. Buchmann, H. Nam-Tran, and U. W. Kesselring, J. Comput.Aided Mol. Des. 6, 431 (1992).

${ }^{19}$ T. Steiner and G. Koellner, J. Mol. Biol. 305, 535 (2001).

${ }^{20}$ J. L. Atwood, F. Hamada, K. D. Robinson, G. W. Orr, and R. L. Vincent, Nature (London) 349, 683 (1991).

${ }^{21}$ T. Steiner, A. M. M. Schreurs, J. A. Kanters, and J. Kroon, Acta Crystallogr., Sect. D: Biol. Crystallogr. 54, 25 (1998).

${ }^{22}$ B. R. T. Simoneit, Geochim. Cosmochim. Acta 57, 3241 (1993).

${ }^{23}$ T. B. Brill, J. Phys. Chem. A 104, 4343 (2000).

${ }^{24}$ J. Gao, J. Am. Chem. Soc. 115, 6893 (1993).
${ }^{25}$ P. T. Cummings, A. A. Chialvo, and H. D. Cochran, Chem. Eng. Sci. 49, 2735 (1994).

${ }^{26}$ J. Zhou, W. Wang, and C. Zhong, Chinese J. Chem. Eng. 9, 196 (2001).

${ }^{27}$ S. Furutaka and S. Ikawa, J. Chem. Phys. 108, 1347 (1998).

${ }^{28}$ S. Furutaka and S. Ikawa, J. Chem. Phys. 108, 5159 (1998).

${ }^{29}$ S. Furutaka and S. Ikawa, J. Chem. Phys. 113, 1942 (2000); 113 8390(E) (2000).

${ }^{30}$ S. Furutaka and S. Ikawa, Fluid Phase Equilib. 185, 379 (2001).

${ }^{31}$ S. Furutaka, H. Kondo, and S. Ikawa, Bull. Chem. Soc. Jpn. 74, 1775 (2001).

${ }^{32}$ J. O. Howell, J. M. Goncalves, C. Amatore, L. Klasinc, R. M. Wightman, and J. K. Kochi, J. Am. Chem. Soc. 106, 3968 (1984).

${ }^{33}$ H. Ratajczak and W. J. Orville-Thomas, J. Mol. Struct. 19, 237 (1973).

${ }^{34}$ R. S. Mulliken, J. Am. Chem. Soc. 74, 811 (1952).

${ }^{35}$ R. M. Bentwood, A. J. Barnes, and W. J. Orville-Thomas, J. Mol. Spectrosc. 84, 391 (1980).

${ }^{36}$ M. P. Conrad and H. L. Strauss, Biophys. J. 48, 117 (1985).

${ }^{37}$ M. P. Conrad and H. L. Strauss, J. Phys. Chem. 91, 1668 (1987).

${ }^{38}$ W. J. Griffiths and F. M. Harris, Org. Mass Spectrom. 22, 559 (1987).

${ }^{39}$ R. S. Mulliken and W. B. Person, Molecular Complexes (WileyInterscience, New York, 1969). 